\title{
Financial communication: Narrative and argument in the pursuit of sustainable trust - Introduction to the Thematic Section
}

Carlo Raimondo*, USI - Università della Svizzera italiana, Switzerland

Andrea Rocci, USI - Università della Svizzera italiana, Switzerland

*Corresponding author: carlo.raimondo@usi.ch

In the emerging field of financial communication, interdisciplinary research based on the analysis of texts and discourse is gaining a prominent role. Bridging quantitative and qualitative approaches and covering financial disclosures, investor relations, business and financial media, as well as the communication of financial intermediaries and regulators, discourse-based studies of financial communication are becoming a unique interdisciplinary crossroads of research developed in accounting, applied linguistics, corporate communication, finance, rhetoric, sociology and natural language processing. This is the main goal of this Thematic Section published in Studies in Communication Sciences and following the second edition of the "Discourse Approaches to Financial Communication" (DAFC) conference held in Lugano in the summer 2017. ${ }^{1}$ While trust has been always recognized as one of the most crucial components of financial interactions, the concrete communication processes by which individual, organizational and inter-organizational trustworthiness is preserved over time or restored after a crisis remain substantially under investigated. This Thematic Section contributes to fill this gap, examining the discursive dynamics of trust in the financial context from different disciplinary and methodological perspectives.

Four relevant papers have been selected for publication. The papers span the communication of central banks, the role of investor relations in creating and main-

1 The conference has been partially funded by the Swiss National Science Foundation: "Discourse Approaches to Financial Communication 2. Building Trust in the Markets with Story and Argument", grant number 10CO1C_174131. taining trust, how trust is enhanced by annual reports, and the relations between trust and corporate social responsibility. The first paper by Laurence Harris (Centre de Recherches Anglophones (CREA), Université Paris Nanterre, France) entitled "In search of 'the Holy Grail of permanent price stability': How the Bank of England communicates trust via story and argument" examines 71 spechees delivered by the Governor of the UK Central Bank spanning the period between the Second World War and the Brexit announcement. The paper sheds light on how, in the communication of central banks, narratives and argument are pivotal in generating and maintaining trust in financial markets in efforts to preserve the stability of the economy. The author identifies and describes the main narrative themes which have been used over the relevant time period. In the following paper by Merja Koskela (University of Vaasa, Department of Communication Studies, Finland) and Belinda Crawford Camiciottoli (University of Pisa, Department of Philology, Literature and Linguistics, Italy) entitled "Different paths from transparency to trust? A comparative analysis of Finnish and Italian listed companies' investor relations communication practices", the focus is shifted on the comparison of investor relations practices between Italy and Finland. The authors, combining a qualitative and a quantitative approach, show how the different cultures do influence the communication towards investors and their effectiveness. For instance, the extraction of statistical keywords from the two corpora shows a clearly different cultural understanding of the requirement of transparency in the communication to investors, while the qualitative rhetorical analysis hints at different 
strategies in leveraging on transparency to foster investors' trust. The third paper of the section is that by Henrik Rahm (Centre for Languages and Literature, Lund University, Sweden), Niklas Sandell (Centre for Languages and Literature, Lund University, Sweden), and Peter Svensson (Centre for Languages and Literature, Lund University, Sweden) entitled "Corporate dreams - Appropriate aspirations and the building of trust in annual reports". The authors shed a new light on how to consider the communication of corporate annual reports. In their view the annual reports are used to convey the corporate dreams, to be intended as "ongoing self-narration of the company, in which it tries to display an allegiance to a set of appropriate aspirations that are considered legitimate in contemporary global capitalism". Thanks to an extensive corpus analysis, the authors show how this phenomenon helps the company to generate external legitimacy and, therefore, to achieve investors' trust. In the final paper of the Thematic Section, entitled "Walmart's communication techniques for sustaining stakeholder trust: How CEOs answer critics and reassure stakeholders in their annual and CSR report letters", Catherine Resche (Foreign language department, University Paris 2 Panthéon-Assas \& CELISO, Sorbonne University, France) focuses on the annualletter-to-shareholders genre and its role in creating, preserving, and, whenever necessary, restoring investor trust. Using a corpus of Walmart's annual letters, the author provides new evidence of how effective proper and timely communication can be to fully address investors' concerns and opponents' attacks.

Overall the four papers contribute to strengthen the discourse-based literature on the importance of communication for different stakeholders in the financial markets, taking the perspective of corporations, investors, central banks, and the general public. In order to do so, they apply an array of rhetorical and corpus linguistic techniques useful to deal with textual data. The main challenge of this relevant, but still fledging field of study will be to be able to communicate relevant research results effectively to all the different disciplines with which it is related. What we have seen in the DAFC 2017 conference and in the articles of this Thematic Section is surely encouraging in going ahead in this direction. 